lich beeinflusst und beeinträchtigt, haben sich Murphy und ihre Kollegen dem Ruf nach mehr Forschung zu diesem Erschöpfungssyndrom angeschlossen. Durch gezielte Befragungen betroffener Patienten wollten sie den Einfluss der Fatigue auf die Lebensqualität, die soziale Teilhabe und die Funktionalität ermitteln.

Insgesamt 267 Patienten wurden an zwei US-amerikanischen Universitäten sowie über Social Media- und Webseiten von SScVereinen für die Studie rekrutiert und befragt. Sie waren im Schnitt 53,7 Jahre alt, überwiegend weiblich (91\%) und hatten die Diagnose im Mittel neun Jahre zuvor erhalten. Die Fragebögen enthielten Fragen zu sozialer Teilhabe (z.B. Aktivitäten mit Familie oder Freunden), körperlicher Funktionalität (z.B. Spaziergänge, Treppensteigen etc.), Lebensqualität, demografischen und klinischen Merkmalen (Alter, Geschlecht, Bildungsstatus, Familienstand etc.) sowie schmerzbedingten Beeinträchtigungen und depressiven Symptomen. Die Fatigue wurde gemessen mit Hilfe des PROMIS-29 (Patient-Reported Outcomes Measurement Information System).

Ihre Gesundheit bewerteten 43,9\% der Patienten insgesamt als mittelmäßig oder schlecht; besonders hoch war der Anteil dieser Patienten bei Overlap-SSc (SSc mit anderen rheumatischen Erkrankungen, $51,6 \%$ ). Die Fatigue empfanden sie dabei als das schlimmste Symptom, gefolgt von den schmerzbedingten Beeinträchtigungen. Patienten mit Overlap-SSc hatten die höchsten Werte für Fatigue und Schlafstörungen.

\section{Fatigue bei Systemischer Sklerose beeinträchtigt die soziale Teilhabe}

\footnotetext{
Murphy SL, Kratz AL, Whibley D et al. Fatigue and Its Association With Social Participation, Functioning, and Quality of Life in Systemic Sclerosis. Arthritis Care \& Research 2021; 73 : 415-422
}

Patienten mit Systemischer Sklerose (SSc) leiden häufig unter Fatigue. Da diese viele Bereiche des Lebens erheb-
Weder Alter noch SSc-Subtyp waren assoziiert mit den Outcome-Messwerten. Fatigue war von allen Outcome-Messwerten am stärksten verbunden mit einer verminderten Fähigkeit zur sozialen Teilhabe; sie allein hatte einen ähnlich starken Einfluss wie schmerzbedingte Beeinträchtigungen und depressive Symptome zusammen genommen. Ein Grund dafür könnte sein, dass Beeinträchtigungen bei der Arbeit in die Beurteilung der sozialen Teilhabe mit einbezogen wurden, denn bei SSc korreliert Fatigue stark mit Arbeitsunfähigkeit. War die Fatigue nur gering ausgeprägt, zeigte sich 
keine Assoziation zwischen diesem Symptom und der sozialen Teilhabe.

\section{FAZIT}

Klinische Assessments beschäftigen sich meist mit den körperlichen Funktionen, doch bei SSc-Patienten ist es wichtig auch die soziale Teilhabe zu berücksichtigen, insbesondere, wenn die Patienten unter starker Fatigue leiden. Dadurch könnten sich neue therapeutische Interventionsmöglichkeiten ergeben, wie z.B. eine Arbeitsplatzanpassung. Außerdem sollten Behandlungen, die auf eine Verbesserung der körperlichen Funktionen und der Lebensqualität abzielen, auch Maßnahmen zur Schmerzlinderung sowie zur Verbesserung depressiver Symptome und Fatigue beinhalten. Vor allem bei Patienten mit Overlap-SSc dürfte die Fatigue ein wichtiger therapeutischer Ansatzpunkt sein.

Stephanie Gräwert, Leipzig 